\title{
Combined CD4 T-Cell and Antibody Response to Human Minor Histocompatibility Antigen DBY After Allogeneic Stem-Cell Transplantation
}

\author{
Fabrice Porcheray ${ }^{1}$, David B. Miklos ${ }^{2}$, Blair H. Floyd ${ }^{1}$, Stefanie Sarantopoulos ${ }^{3}$, Roberto \\ Bellucci $^{1}$, Robert J. Soiffer ${ }^{1}$, Joseph H. Antin ${ }^{1}$, Edwin P. Alyea ${ }^{1}$, Jerome Ritz ${ }^{1}$, and \\ Emmanuel Zorn ${ }^{1,4}$ \\ ${ }^{1}$ Currently, Department of Surgery, Transplant Center, Massachusetts General Hospital, Harvard \\ Medical School, Boston, MA; formerly, Department of Medical Oncology, Dana-Farber Cancer \\ Institute, Harvard Medical School, Boston, MA. \\ ${ }^{2}$ Department of Medicine, Stanford University Medical Center, Stanford, CA. \\ ${ }^{3}$ Division of Hematology/Oncology, Lineberger Comprehensive Cancer Center, University of \\ North Carolina Medical Center, Chapel Hill, NC.
}

\begin{abstract}
Background-Antibody responses to HY antigens in male recipients are frequent after transplantation of stem cells from female donors (Miklos et al., Blood 2005; 105: 2973; Miklos et al., Blood 2004; 103: 353). However, evidence that this B-cell immunity is accompanied by T-cell responses to the cognate antigens is scarce. Here, we examined T- and B-cell responses to DBY antigen in a male patient who received hematopoietic stem cells from a human leukocyte antigenidentical female sibling.
\end{abstract}

Materials and Methods-We used 93 overlapping peptides representing the entire DBY protein to detect and characterize T-cell and antibody responses to DBY by enzyme-linked immunosorbent spot (ELISPOT) and enzyme-linked immunosorbent assay.

Results-High frequency CD4+ T cells specific for a unique DBY peptide were detected in the patient blood. We isolated the corresponding T-cell clone and characterized the recognized epitope as an 18-mer peptide restricted by human leukocyte antigen-DRB $1 * 0101$. Upon stimulation, this clone produced cytokines with no evidence of Th1 or Th2 polarization. Remarkably, this clone also recognized the DBX homologue peptide and responded to female donor dendritic cells stimulated with poly $\mathrm{I} / \mathrm{C}$ or lipopolysaccharide, indicating that the peptide was endogenously processed in these cells. High titer DBY-specific antibodies were also found in the patient serum which, in contrast to the T-cell response, did not cross-react with DBX.

Conclusion-We show here the development of a coordinated B and T-cell response to DBY in a recipient of sex mismatched allogeneic hematopoietic stem-cell transplantation. Our findings support a role for CD4+ T cells in the development of humoral immunity to minor histocompatibility antigens.

\footnotetext{
Copyright (C) 2011 by Lippincott Williams \& Wilkins

${ }^{4}$ Address correspondence to: Emmanuel Zorn, Ph.D., Massachusetts General Hospital, Wellman-Thier 815, 55 Fruit Street, Boston, MA 02114. ezorn@partners.org.

The authors declare no conflict of interest.

Supplemental digital content is available for this article. Direct URL citations appear in the printed text, and links to the digital files are provided in the HTML text of this article on the journal's Web site (www.transplantjournal.com).
} 


\section{Keywords}

Hematopoietic stem-cell transplantation; H-Y antigens; Antibodies; CD4+ T cells

Studies from our laboratory have shown that sex-linked minor histocompatibility antigens $(\mathrm{H}-\mathrm{Y})$ elicit isotype switched antibody responses in approximately $50 \%$ of male recipients of allogeneic hematopoietic stem-cell transplants (HSCT) from female donors (1, 2). H-Y antigens constitute a distinct class of minor histocompatibility antigen (mHA) encoded by ubiquitously expressed male-specific genes located in the nonrecombining region of the $\mathrm{Y}$ chromosome (3). These genes are significantly disparate from their homologues located on the X chromosome. At the amino acid level, Y and X gene products show between $91 \%$ and 99\% identity (4-11). Upon transplantation into male patients, female donor $\mathrm{T}$ cells elicit a strong immune response directed to H-Y mHAs. H-Y-specific antibodies are associated with a higher incidence of chronic graft versus host disease (cGVHD) and lower risk of tumor relapse, suggesting a role in both graft versus host $(\mathrm{GVH})$ and graft versus leukemia (GVL) effects (1). It is presumed that an important component of humoral immunity is directed toward allogeneic targets such as $\mathrm{H}-\mathrm{Y}$ antigens. In humans, the conditions leading to the development of such allogeneic antibody responses are still largely unknown. B-cell class switch recombination is presumed to require help from CD4+ T cells specific for the cognate antigens. We report here the comprehensive characterization of a coordinated Tand B-cell response to $\mathrm{H}-\mathrm{Y}$ antigen DBY in a male recipient of human leukocyte antigen (HLA)-identical female HSCT.

\section{RESULTS}

\section{DBY mHA Elicits a Sustained T-cell Response After HSCT}

Ninety-three overlapping peptides representing the entire amino acid sequence of DBY were synthesized and distributed in 12 pools. Peripheral blood mononuclear cells (PBMC) collected from a male patient 31 months after receiving allogeneic stem cells from a female donor were assessed for reactivity to each peptide pool using an interferon (IFN)- $\gamma$ enzymelinked immunosorbent spot (ELISPOT) assay. To avoid skewing the representation of potential DBY-reactive cells, ELISPOT assays were performed using unmanipulated PBMC, without previous in vitro sensitization or expansion. A high level of reactivity was observed towards one DBY peptide pool (data not shown). Testing of individual peptides included in this pool assigned the reactivity to a single peptide, DBY 427-444 (Fig. 1A). The frequency of IFN- $\gamma$ producing T cells specific to this DBY epitope was approximately $1.5 \times 10^{-4}$. We cannot rule out that additional DBY reactive T-cell clones secreting other cytokines than IFN $\gamma$ were also present in the patient blood. The high frequency T-cell reactivity against DBY 427-444 was observed in PBMC collected at various times after HSCT. As shown in Figure 1(B), the T-cell response was sustained between 18 and 38 months after transplant.

\section{The DBY-Specific CD4+ T-Cell Response Is Restricted by HLA-DRB1 0101}

ELISPOT experiments using PBMC depleted of CD4+ or CD8+ T cells revealed that DBY peptide-reactive cells were included in the CD4+ T-cell subset (data not shown). One CD3+CD4+ T-cell clone (clone 42; Fig. 2A) displayed the highest reactivity to donor B cells immortalized with Epstein-Barr virus (EBV-B) pulsed with DBY 427-444 and was expanded in vitro for further studies. T-cell receptor (TCR) V $\beta$ repertoire analysis performed using RNA extracted from clone 42 cells demonstrated clonality and revealed the usage of the TCRVB2 segment (data not shown). Flow cytometry analysis using monoclonal antibodies (mAbs) specific to TCRVB2confirmed the clonality of clone 42 (Fig. 2B). The 
specificity of clone 42 for DBY peptide 427-444, but not overlapping peptides, was determined by IFN- $\gamma$ enzyme-linked immunosorbent assay (ELISA) using donor EBV-B cells pulsed with different concentrations of peptides as target cells (Fig. 2C).

As shown in Figure 2(D), stimulation of clone 42 with EBV B cells pulsed with DBY 427444 was inhibited by anti-HLA-DR mAbs, but not by anti-HLA class I antibodies. These results suggested that TCR recognition was restricted by HLA-DR. Likewise, clone 42 reactivity was inhibited by a blocking $\mathrm{mAb}$ specific for TCRVB2 but not by a control $\mathrm{mAb}$ specific for the TCRVB3 variable region. To determine which HLA-DR allele was recognized by clone 42 , we used a series of third-party allogeneic EBV B cells expressing one of the two HLA-DR alleles expressed by both patient and donor: HLA-DRB $1 * 1101$ and HLA-DRB $1 * 0101$. As shown in Figure 2(E), cells expressing DRB $1 * 0101$ stimulated clone 42 when pulsed with the antigenic DBY peptide, whereas cells expressing DRB $1 * 1101$ were not recognized. These experiments demonstrated that presentation of the DBY 427-444 peptide to clone 42 was restricted by HLA-DRB $1 * 0101$. Analysis of the DBY amino acid sequence using the SYFPEITHI epitope prediction algorithm attributed the highest score and first rank to the antigenic peptide starting in position 427 compared with all other peptides (see Table, Supplemental Digital Content 1, http://links.lww.com/TP/A438). This analysis further confirmed the high affinity of peptide DBY 427-444 for the HLA-DRB $1 * 0101$ groove.

\section{Both DBX and DBY Peptides Are Recognized by Clone $\mathbf{4 2}$}

We then tested whether clone 42 would also recognize the corresponding X-encoded peptide, DBX 429-446, which differs from DBY 427-444 by three residues (Fig. 3A). Despite these disparate amino acids, both $\mathrm{X}$ and $\mathrm{Y}$ peptides stimulated clone 42 at a similar level when pulsed on donor EBV B cells (Fig. 3A).

\section{T-Cell Clone 42 Recognizes Endogenously Processed DBX Antigen in Stimulated Female Donor Dendritic Cells}

We determined whether clone 42 could recognize endogenously processed DBX protein in female donor dendritic cells (DCs). Clone 42 was tested for its capacity to react to the female donor DCs either unstimulated or stimulated with lipopolysaccharide (LPS) or polyI/C. As shown in Figure 3(B), stimulated DCs were recognized by clone 42, indicating that DBX peptide was processed and presented to the T-cell clone. The clone stimulation was blocked by anti-HLA-DR mAb.

\section{Clone 42 Produces Both Th1 and Th2-Type Cytokines}

Upon stimulation with peptide DBY 427-444, clone 42 produced a wide range of cytokines (Fig. 4), including the pro-inflammatory Th1-type cytokines interleukin (IL)-8, IFN- $\gamma$, and tumor necrosis factor $\alpha$. Anti-inflammatory cytokines (Th2) IL-4, IL-13, and IL-10 were also produced. Clone 42 did not appear to secrete IL-17, TGF- $\alpha$, or IFN- $\alpha$.

\section{Development of an Antibody Response Specific to DBY}

We investigated whether an antibody response to DBY and DBX antigens had also developed after allogeneic transplantation in this patient. As shown in Figure 5(A), patient serum collected 24 months after HSCT strongly reacted with recombinant DBY protein by Western Blot assays. In contrast to the T-cell response, which did not differentiate between DBX and DBY epitopes, DBY antibodies did not react with DBX. We then used 93 synthetic overlapping DBY peptides in ELISA assays to map the patient's antibody response and identify the corresponding antigenic epitopes. As illustrated in Figure 5(B), the serum reacted with three peptides: DBY 7-25, DBY 104-122, and DBY 112-138. Even though 
antibody binding to peptides may not fully reflect binding to the native protein, it is remarkable that the three peptide epitopes are located in regions of the protein that are the most disparate between DBY and DBX.

We then used ELISA to assess the reactivity of serial serum samples collected at various times after HSCT to the three most antigenic DBY peptides and their DBX counterparts. Results reported in Figure 5(C) reveal the development of antibodies reactive to the DBY peptides but not to the corresponding DBX peptides. The serologic response developed at 8 to 14 months after transplantation and reached a plateau between 14 and 27 months posttransplant. Reactivity to these peptides started to decrease after 27 months posttransplant and became virtually undetectable at 5 years postbone marrow transplantation. No reactivity was detected before or 6 months after transplant, indicating that the antibody response developed relatively late after HSCT.

\section{DISCUSSION}

Previous studies from our laboratory have shown that H-Y mHAs also elicit isotype switched IgG antibody responses after allogeneic $\operatorname{HSCT}(2,12)$. We demonstrated that approximately $50 \%$ of male patients who received HSCT from female donors developed high titer antibody responses to DBY (2). The development of antibodies to $\mathrm{H}-\mathrm{Y}$ correlated with the development of cGVHD and disease remission, suggesting a role in both GVH and GVL effects (1).

In addition, a number of clinical trials using rituximab have shown clinical improvement in patients with steroid-refractory chronic graft-versus-host disease (for review [13]). One trial performed at our institution resulted in clinical improvement in $70 \%$ of the patients, which was associated with a decrease in anti-HY antibodies. These studies (review in [14]) highlighted the relevance of B-cell immunity after allogeneic HSCT. How these antibody responses develop amid immune reconstitution in HSCT recipients remains obscure. Class switch recombination, leading to the generation of antigen-specific IgG antibodies, is presumed to require help from cognate antigen specific CD4+ T cells. To confirm this point, we used a strategy aimed at detecting high frequency T-cell responses to DBY, a prototypic $\mathrm{H}-\mathrm{Y}$ antigen. As previously described, this approach consists in assessing the reactivity of blood cells ex vivo to DBY without previous in vitro presensitization (12). In addition to the high frequency T-cell response, the patients' serum also revealed the presence of $\operatorname{IgG}$ specific for DBY. Collectively, these observations support the hypothesis that antibodies to $\mathrm{H}-\mathrm{Y}$ develop with the help of cognate antigen-specific CD4+ T cells. Although DBY is not expressed on the cell surface, this protein is expressed in many tissues. Anti-DBY antibodies may enhance the uptake of this antigen by antigen presenting cell and its presentation to $T$ cells through the formation of immune complexes. These antibodies would thus participate and exacerbate the immune response after tissue destruction.

Remarkably, CD4 T-cell clones reacted with both DBY and DBX epitopes, whereas antibodies were specific for DBY and did not cross-react with DBX. These findings reveal a complex autoimmune T-cell and alloimmune B-cell response, sharing a number of characteristics with the anti-H-Y immune response that developed in another female to male HSCT recipient (12). In the previous report, coordinated T- and B-cell responses were described in a context of cGVHD, whereas in our present study these responses do not appear to be associated with GVH. The two CD4+ T-cell responses were restricted by HLADRB1*1501 and HLA-DRB $1 * 0101$, respectively. The sequences of the two recognized DBY peptides show minimal homology. Both clones recognize DBY and DBX peptides and both peptides are located in regions showing disparate amino acids between DBY and DBX. DBY and DBX peptides differed by two amino acids in our previous report and by three 
amino acids in the current report. Further studies are needed to accurately define the prevalence of such mHA-specific T cells after allogeneic HSCT and the extent to which T cells with this pattern of reactivity contribute to both GVHD and GVL.

The presence of alloantibodies is associated with chronic GVHD and protection from disease relapse after HSCT. Nevertheless, cases of transplant recipients with high antibody titers to H-Y antigens without any manifestation of chronic GVHD have also been observed, indicating that antibodies alone are not sufficient to induce damage to normal tissues after HSCT. In the present study, the development of the antibody response and presence of T cells specific to DBY coincided with interstitial pneumonitis, which was likely related to a GVH reaction. Remarkably, high titer IgG and high frequency CD4+ T cells specific to DBY persisted long after the clinical episode, at a time when the patient had no apparent manifestations of GVHD. Even though the clinical incidence of anti-DBY antibodies can not be determined from this work, our study suggests that anti-DBY antibodies may not necessarily be detrimental but could also contribute to establishing long-term tolerance to the recipient. Alloantibodies have previously been associated with tolerance after solid organ transplantation or combined kidney-bone marrow transplant (15). Likewise, the sole detection of CD4+ T cells specific for DBY in the blood may not be sufficient to appreciate their role in vivo. Allospecific Tregs have also been described in mice and humans. In the absence of chronic GVHD in this patient, it is tempting to speculate that DBY-specific T cells may have had a regulatory function. A cytokine profile analysis of the DBY specific clone did not reveal any clear pattern. Clone 42 secretes both Th1 and Th2 type cytokines but did not produce IL-17.

Overall, our study provides additional evidence that H-Y mHA can elicit complex immune responses combining isotype switched antibodies and specificCD4+ T cells in male recipients of female HSCT. However, observing such immunity in the absence of GVHD, suggests that $\mathrm{H}-\mathrm{Y}$ specific immune cells and antibodies may not be pathogenic and may even participate in tolerance mechanisms after HLA identical HSCT.

\section{MATERIALS AND METHODS}

\section{Patient Characteristics}

The patient studied in this report is a 63-year-old man with myelodysplastic syndrome who received an allogeneic HSCT from his HLA identical sister. The patient received a reduced intensity conditioning regimen before HSCT consisting of fludarabine and busulfan $(16,17)$. The patient received cyclosporine and prednisone as prophylaxis for GVHD and engraftment was achieved at day +7 . The patient developed significant acute GVHD of the gut 2 months after HSCT that was successfully treated with increased prednisone, tacrolimus, and mycophenolate mofetil. At 16 months posttransplant, the patient developed interstitial pneumonitis possibly related to chronic GVHD. No causative pathogen was identified. He was successfully treated with prednisone for a period of 4 months when symptoms resolved. The patient currently remains disease-free 10 years posttransplant without clinical evidence of ongoing chronic GVHD and has a normal white blood count.

\section{Peptides}

Synthetic peptides were obtained from New England Peptides (Gardner, MA). Peptides were crude (93 screening peptides; purity $\sim 70 \%$ ), or more than $85 \%$ purity (DBY420-437, DBY427-444, DBY434-450, DBX201-218, DBX429-446, DBY7-25, DBX7-25, DBY104-122, DBX104-123, DBY112-128, and DBX114-130). Each peptide was reconstituted at $10 \mathrm{mg} / \mathrm{mL}$ in DMSO and stored at $-20^{\circ} \mathrm{C}$. 


\section{ELISPOT Assays}

ELISPOT assays were performed as previously described (18).

\section{Cell Culture}

T-cell clones were cultured in Roswell Park Memorial Institute (RPMI) 1640 supplemented with $10 \%$ heat-inactivated human AB serum, glutamine, sodium pyruvate, Hepes, and antibiotics (referred to as T-cell medium) in the presence of $100 \mathrm{U} / \mathrm{mL}$ recombinant IL-2. Medium was replenished twice weekly with fresh IL-2. Immature DCs were obtained as previously described (19). In brief, 40 to $60 \times 10^{6} \mathrm{PBMCs}$ were incubated for $15 \mathrm{~min}$ in a 75 $\mathrm{cm}^{2}$ culture flask (Corning Inc.). Non-adherent cells were removed by thoroughly washing the flask three times with medium. The remaining adherent cells were incubated for 1 week in medium supplemented with $1000 \mathrm{U} / \mathrm{mL}$ IL-4 (R\&D Systems) and $1000 \mathrm{U} / \mathrm{mL}$ granulocyte macrophage colony-stimulating factor (GM-CSF) (R\&D Systems). Cells were then harvested, washed, and used as immature DCs. Maturation of DCs was achieved by incubating cells for $24 \mathrm{hr}$ with $25 \mathrm{ng} / \mathrm{mL}$ polyinosinic-polycytidylic acid (Amersham Biosciences) or $100 \mathrm{ng} / \mathrm{mL}$ LPS (Sigma-Aldrich).

\section{Generation of a DBY-Reactive T-Cell Clone}

A T-cell line was first generated by stimulating $6 \times 10^{6} \mathrm{PBMC}$ collected from the patient 31 months after HSCT in the presence of peptide DBY 427-444 at a final concentration of 25 $\mu \mathrm{g} / \mathrm{mL}$ in the presence of $100 \mathrm{U} / \mathrm{mL}$ IL-2. One week after stimulation, CD4+ T cells were immunopurified from the cell line using anti-CD4+ microbeads (Miltenyi Biotec, Auburn, $\mathrm{CA})$ and subsequently cloned by limiting dilution on feeder cells consisting of irradiated patient EBV B cells $\left(1.5 \times 10^{4}\right.$ cells/well; $\left.60 \mathrm{~Gy}\right)$ and allogeneic PBMC $\left(7 \times 10^{4}\right.$ cells/well; $35 \mathrm{~Gy}$ ) in the presence of $100 \mathrm{U} / \mathrm{mL}$ recombinant human IL-2. All of the T-cell clones generated were assessed for specific reactivity with the DBY peptide. Clone 42 displayed the strongest reactivity and was further expanded under the same culture conditions with the addition of anti-CD3 mAb (clone OKT3, eBioscience, San Diego CA) at a concentration of $30 \mathrm{ng} / \mathrm{mL}$.

\section{IFN-y Secretion Assays}

IFN- $\gamma$ secretion by T-cell clone 42 was assessed by incubating $5 \times 10^{4}$ responder cells in 96well, U-bottom microplates with $5 \times 10^{4}$ stimulator cells in $200 \mu \mathrm{L}$ T-cell medium containing $50 \mathrm{U} / \mathrm{mL}$ IL-2. Stimulator cells consisted of autologous or third-party allogeneic EBV B-cells pulsed with synthetic peptides $(10 \mu \mathrm{g} / \mathrm{mL}$ or as otherwise stated) for $1 \mathrm{hr}$ at $37^{\circ} \mathrm{C}$ and washed twice to remove excess peptide. Autologous cells were prepared from the patient' blood when hematopoietic chimerism was $100 \%$. After $18 \mathrm{hr}$ of incubation, $50 \mu \mathrm{L} /$ well supernatant was harvested and IFN- $\gamma$ was measured using an ELISA kit (Pierce Endogen, Rockford, IL) according to the manufacturer's instructions. In experiments with DCs, 10,000 immature DCs/well were incubated in $100 \mu \mathrm{L}$ medium with or without LPS or poly-I/C for $24 \mathrm{hr}$ at $37^{\circ} \mathrm{C}$. A total of $4 \times 10^{4}$ clone 42 cells were then added to the culture in

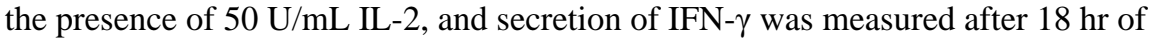
incubation.

\section{Flow Cytometry}

Clone 42 was labeled with an fluorescein isothiocyanate-conjugated anti-TRBV2 $\mathrm{mAb}$ (clone MPB2D5, Beckman Coulter, Fullerton, CA), or a fluorescein isothiocyanateconjugated isotype control antibody (mouse IgG1, Beckman Coulter). Cells were analyzed using a FC500 flow cytometer (Beckman Coulter). 


\section{Clone 42 Cytokine Production Profiling}

The T-cell clone 42 cytokine secretion pattern was determined by stimulating $5 \times 10^{4}$ cells per well in 96 well U-bottom plate with $5 \times 10^{4}$ autologous EBV B-cells pulsed with DBY peptide 427-444 or control peptide (DBX 201-218) at a concentration of $10 \mu \mathrm{g} / \mathrm{mL}$ for 24 hr. G-CSF, IL-2, IL-4, IL-6, IL-8, IL-9, IL-10, IL-13, IL-17, TGF- $\alpha$, IFN- $\alpha$, IFN- $\gamma$, and tumor necrosis factor $\alpha$ were quantified in the culture supernatants using a custom multiplex ELISA (Pierce SearchLight).

\section{Epitope Prediction for HLA-DRB1*0101}

Using the SYFPEITHI algorithm (http://www.syfpeithi.de/), we defined the "ligation strength" of all 15-mer peptides throughout the DBY sequence to HLA-DRB1*0101. Each peptide is given a score based on its affinity for the HLA molecule as calculated by the algorithm. The table in Supplemental Digital Content 1 (http://links.lww.com/TP/A438) displays ranking and starting positions of DBY peptides with the highest affinity for HLADRB $1 * 0101$.

\section{Recombinant Protein Production and Western Blot Assays}

Recombinant DBX and DBY protein were produced and purified as described (2).

\section{DBY ELISA Assays}

Antibodies to DBX and DBY peptides were measured by ELISA as previously described (2).

\section{Supplementary Material}

Refer to Web version on PubMed Central for supplementary material.

\section{Acknowledgments}

This work was supported by NIH grants CA142106 and AI29530.

E.Z. was the principal investigator and takes primary responsibility for the manuscript; F.P., D.B.M., and B.H.F. performed the laboratory work for this study; S.S. and R.B. participated in data analysis; R.J.S., J.H.A., and E.P.A. recruited the patients and provided clinical data; E.Z. and J.R. coordinated the research; F.P. and E.Z. wrote the manuscript; and other authors reviewed and edited the manuscript.

\section{REFERENCES}

1. Miklos DB, Kim HT, Miller KH, et al. Antibody responses to H-Y minor histocompatibility antigens correlate with chronic graft-versus-host disease and disease remission. Blood. 2005; 105:2973. [PubMed: 15613541]

2. Miklos DB, Kim HT, Zorn E, et al. Antibody response to DBY minor histocompatibility antigen is induced after allogeneic stem cell transplantation and in healthy female donors. Blood. 2004; 103:353. [PubMed: 14512314]

3. Simpson E, Scott D, Chandler P. The male-specific histocompatibility antigen, H-Y: A history of transplantation, immune response genes, sex determination and expression cloning. Annu Rev Immunol. 1997; 15:39. [PubMed: 9143681]

4. Spierings E, Vermeulen CJ, Vogt MH, et al. Identification of HLA class II-restricted H-Y-specific T-helper epitope evoking CD4+ T-helper cells in H-Y-mismatched transplantation. Lancet. 2003; 362:610. [PubMed: 12944060]

5. Warren EH, Gavin MA, Simpson E, et al. The human UTY gene encodes a novel HLA-B8restricted H-Y antigen. J Immunol. 2000; 164:2807. [PubMed: 10679124] 
6. Wang W, Meadows LR, den Haan JM, et al. Human H-Y: A male-specific histocompatibility antigen derived from the SMCY protein. Science. 1995; 269:1588. [PubMed: 7667640]

7. Vogt MH, de Paus RA, Voogt PJ, et al. DFFRY codes for a new human male-specific minor transplantation antigen involved in bone marrow graft rejection. Blood. 2000; 95:1100. [PubMed: 10648428]

8. Vogt MH, Goulmy E, Kloosterboer FM, et al. UTY gene codes for an HLA-B60-restricted human male-specific minor histocompatibility antigen involved in stem cell graft rejection: Characterization of the critical polymorphic amino acid residues for T-cell recognition. Blood. 2000; 96:3126. [PubMed: 11049993]

9. Vogt MH, van den Muijsenberg JW, Goulmy E, et al. The DBY gene codes for an HLA-DQ5restricted human male-specific minor histocompatibility antigen involved in graft-versus-host disease. Blood. 2002; 99:3027. [PubMed: 11929796]

10. Pierce RA, Field ED, den Haan JM, et al. Cutting edge: The HLA-A*0101-restricted HY minor histocompatibility antigen originates from DFFRY and contains a cysteinylated cysteine residue as identified by a novel mass spectrometric technique. J Immunol. 1999; 163:6360. [PubMed: 10586024]

11. Meadows L, Wang W, den Haan JM, et al. The HLA-A*0201-restricted H-Y antigen contains a posttranslationally modified cysteine that significantly affects T cell recognition. Immunity. 1997; 6:273. [PubMed: 9075928]

12. Zorn E, Miklos DB, Floyd BH, et al. Minor histocompatibility antigen DBY elicits a coordinated B and T cell response after allogeneic stem cell transplantation. J Exp Med. 2004; 199:1133. [PubMed: 15096539]

13. Kharfan-Dabaja MA, Mhaskar AR, Djulbegovic B, et al. Efficacy of rituximab in the setting of steroid-refractory chronic graft-versus-host disease: A systematic review and meta-analysis. Biol Blood Marrow Transplant. 2009; 15:1005. [PubMed: 19660713]

14. Perruche S, Kleinclauss F, Tiberghien P, et al. B cell allogeneic responses after hematopoietic cell transplantation: Is it time to address this issue? Transplantation. 2005; 79 suppl 3:S37. [PubMed: 15699746]

15. Porcheray F, Wong W, Saidman SL, et al. B-cell immunity in the context of T-cell tolerance after combined kidney and bone marrow transplantation in humans. Am J Transplant. 2009; 9:2126. [PubMed: 19624570]

16. Alyea EP, Kim HT, Ho V, et al. Impact of conditioning regimen intensity on outcome of allogeneic hematopoietic cell transplantation for advanced acute myelogenous leukemia and myelodysplastic syndrome. Biol Blood Marrow Transplant. 2006; 12:1047. [PubMed: 17067911]

17. Alyea EP, Kim HT, Ho V, et al. Comparative outcome of nonmyeloablative and myeloablative allogeneic hematopoietic cell transplantation for patients older than 50 years of age. Blood. 2005; 105:1810. [PubMed: 15459007]

18. Currier JR, Kuta EG, Turk E, et al. A panel of MHC class I restricted viral peptides for use as a quality control for vaccine trial ELISPOT assays. J Immunol Methods. 2002; 260:157. [PubMed: 11792386]

19. Sallusto F, Lanzavecchia A. Efficient presentation of soluble antigen by cultured human dendritic cells is maintained by granulocyte/macrophage colony-stimulating factor plus interleukin 4 and downregulated by tumor necrosis factor alpha. J Exp Med. 1994; 179:1109. [PubMed: 8145033] 


\section{A Stimulating peptide}
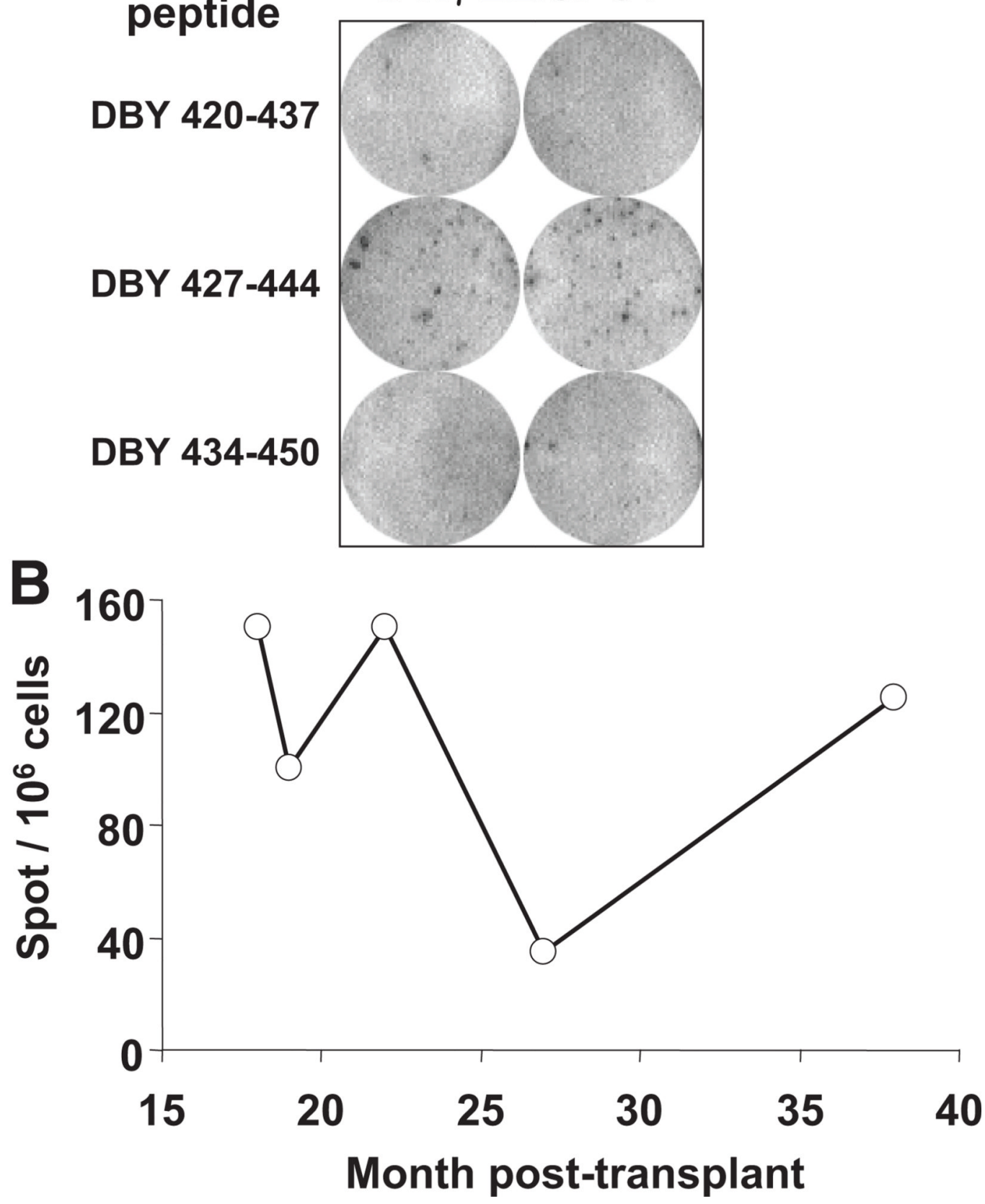

FIGURE 1.

Sustained CD4+ T-cell response to DBY after allogeneic hematopoietic stem-cell transplantation (HSCT). (A) ELISPOT assays for IFN- $\gamma$ secretion were conducted using ex vivo peripheral blood mononuclear cells(PBMC)collected 31 months after HSCT stimulated with overlapping DBY peptides. (B) ELISPOT assays for IFN- $\gamma$ secretion were carried out using ex vivo PBMC collected serially between 18 and 38 months after HSCT stimulated with DBY 427-444. Results are expressed as number of spots per $10^{6}$ cells. 
A

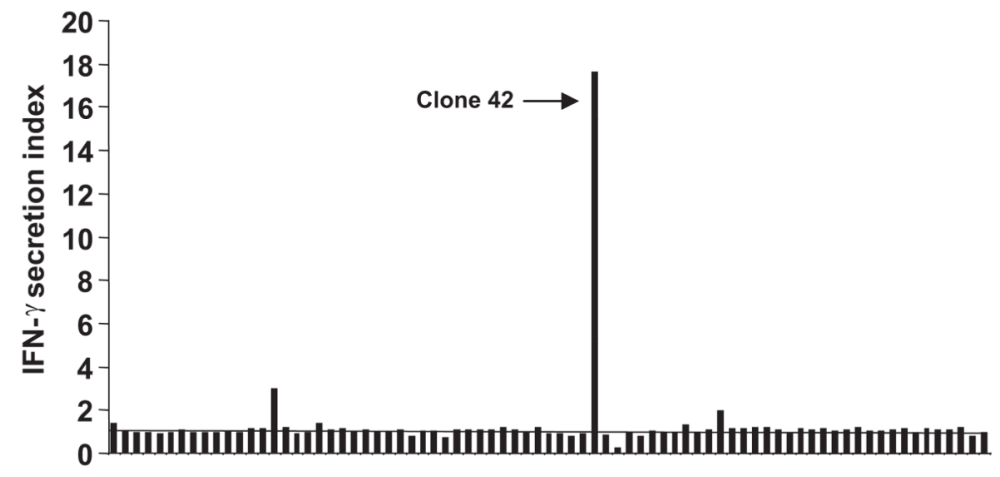

B

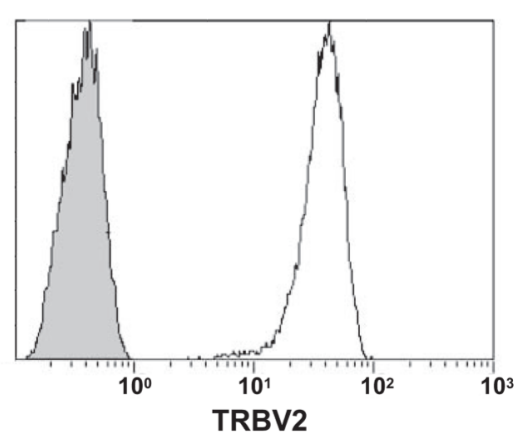

T Cell clones

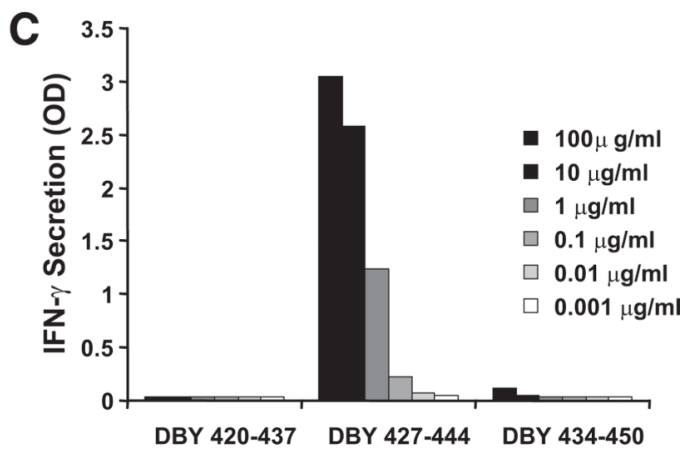

D

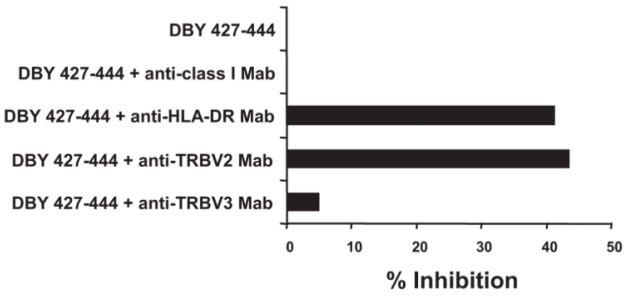

E

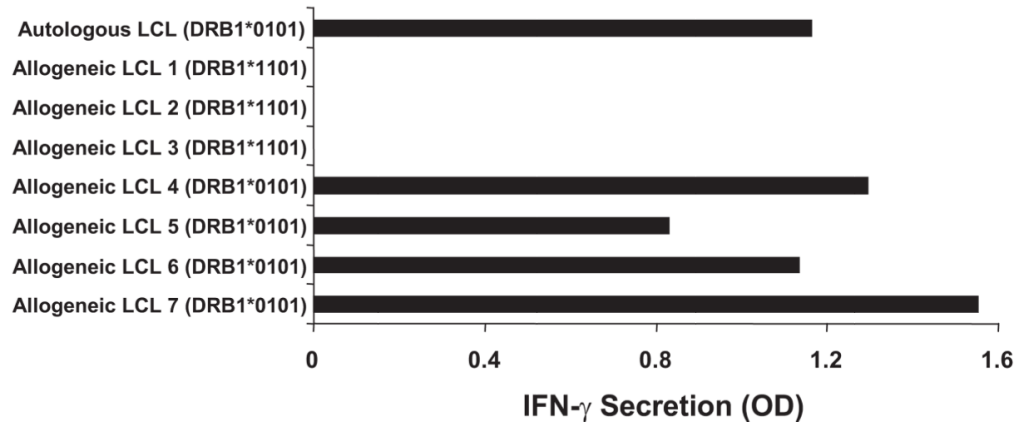

FIGURE 2.

The CD4+ T-cell response to DBY 427-444 is restricted by human leukocyte antigen (HLA)-DRB1*0101 molecules. (A) CD4+ T-cell clones established from the patient blood collected 31 months posttransplant were stimulated with donor Epstein-Barr virus (EBV) Bcells pulsed with peptide DBY 427-444. IFN- $\gamma$ secretion was measured after overnight culture. Clone 42 displayed the high reactivity towards the antigenic peptide. (B) Flow cytometry analysis of clone 42 stained with an anti-TRBV2 monoclonal antibody (white histogram) or with an isotype control antibody (gray histogram). (C) The reactivity of clone 42 stimulated with increasing doses of peptide DBY 427-444 or 2 overlapping 18-mer DBY peptides was measured by IFN- $\gamma$ secretion. (D) CD4+ T-cell clone 42 stimulation with 
autologous EBV-immortalized B cells (autologous cells were prepared from the patient' blood when hematopoietic chimerism was 100\%) pulsed with DBY peptide 427-444 or a control peptide (DBX 201-218) was assessed by measuring IFN- $\gamma$ secretion in the culture supernatant. On the target side, antigen recognition was blocked with anti-HLA-DR mAb (G46.6, BD biosciences) but not anti-HLA class I mAb (W6/32, Sigma Aldrich). On the Tcell side, antigen recognition was blocked with anti-TCRB2 mAb but not with anti-TCRB3 $\mathrm{mAb}$. (E) Clone 42 was stimulated with a series of third-party allogeneic Epstein-Barr virus (EBV)-B cells (LCL) sharing the expression of HLA-DRB1*0101 or HLA-DB1*1101 with the patient after pulsing with DBY 427-444. IFN- $\gamma$ secretion was measured in the culture supernatant after overnight incubation. 
A

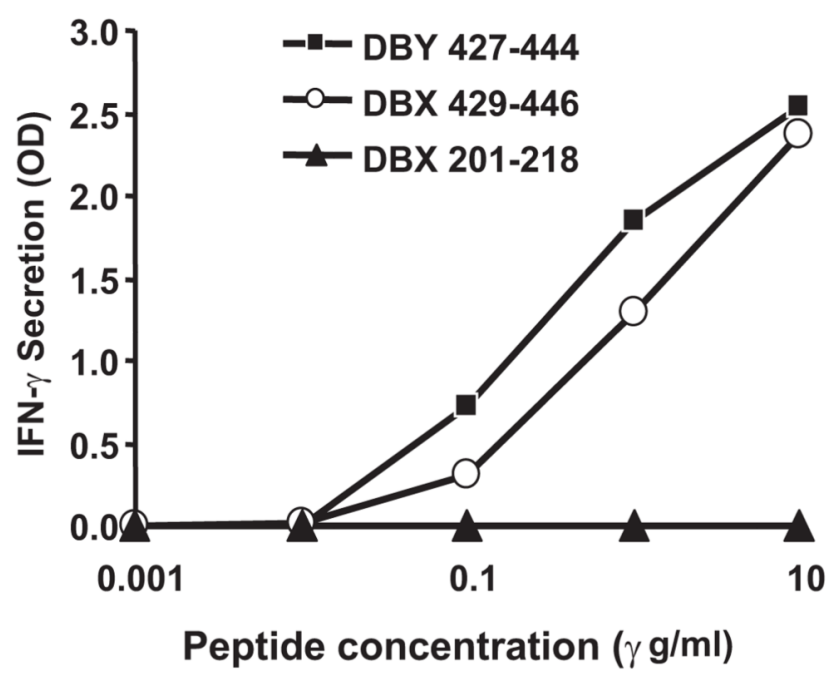

DBY 427-444: SFLLDILGATGSDSLTLV

DBX 429-446: SFLLDLLNATGKDSLTLV

B Stimulator cells:

Female donor DC/Medium

Female donor DC/LPS

Female donor DC/Poly I/C

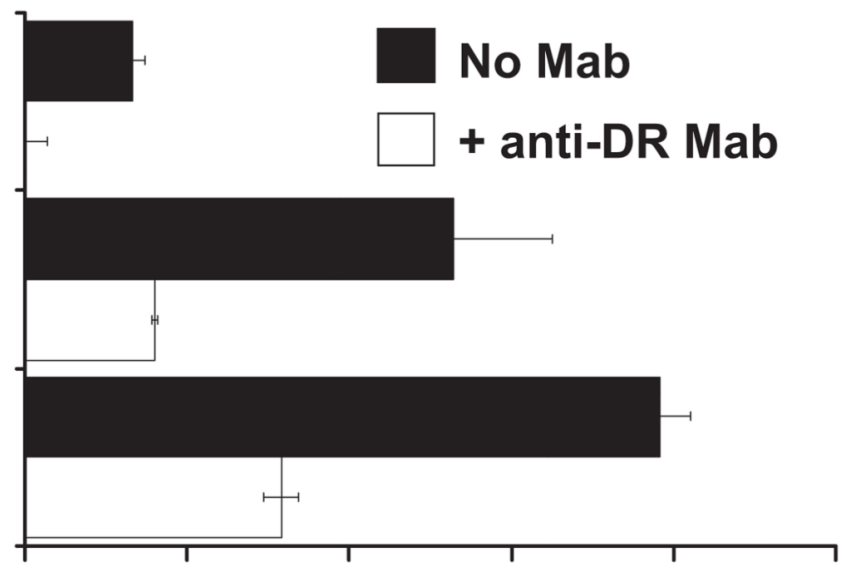

$\begin{array}{llllll}0 & 0.1 & 0.2 & 0.3 & 0.4 & 0.5\end{array}$

IFN- $\gamma$ secretion (OD)

FIGURE 3.

T-cell clone 42 recognizes both DBX and DBY antigens epitopes and DBX antigens endogenously processed and presented by mature dendritic cells (DCs). (A) Clone 42 was stimulated with autologous Epstein-Barr virus (EBV)-B cells pulsed with increasing concentrations of DBY 427-444 peptide and its DBX homologue DBX 429-446. IFN- $\gamma$ secretion was measured in the culture supernatant after overnight incubation. Reactivity to a control peptide, DBX 201-218, is also shown. Disparate residues between DBX and DBY versions of the peptides are represented with underlined characters. (B) Patient' (female) DCs $\left(10^{4} /\right.$ well) were incubated for $24 \mathrm{hr}$ with medium alone, poly-I/C, or lipopolysaccharide (LPS) to induce maturation. T-cell clone $42\left(10^{4} /\right.$ well) was then added to the wells in the 
presence of IL-2 (50 U/mL). After $18 \mathrm{hr}$ of incubation, secretion of IFN- $\gamma$ was determined in cocultures by ELISA. Same assay was run simultaneously in the presence of anti-human leukocyte antigen (HLA)-DR mAb to block antigen presentation to the CD4 clone. 


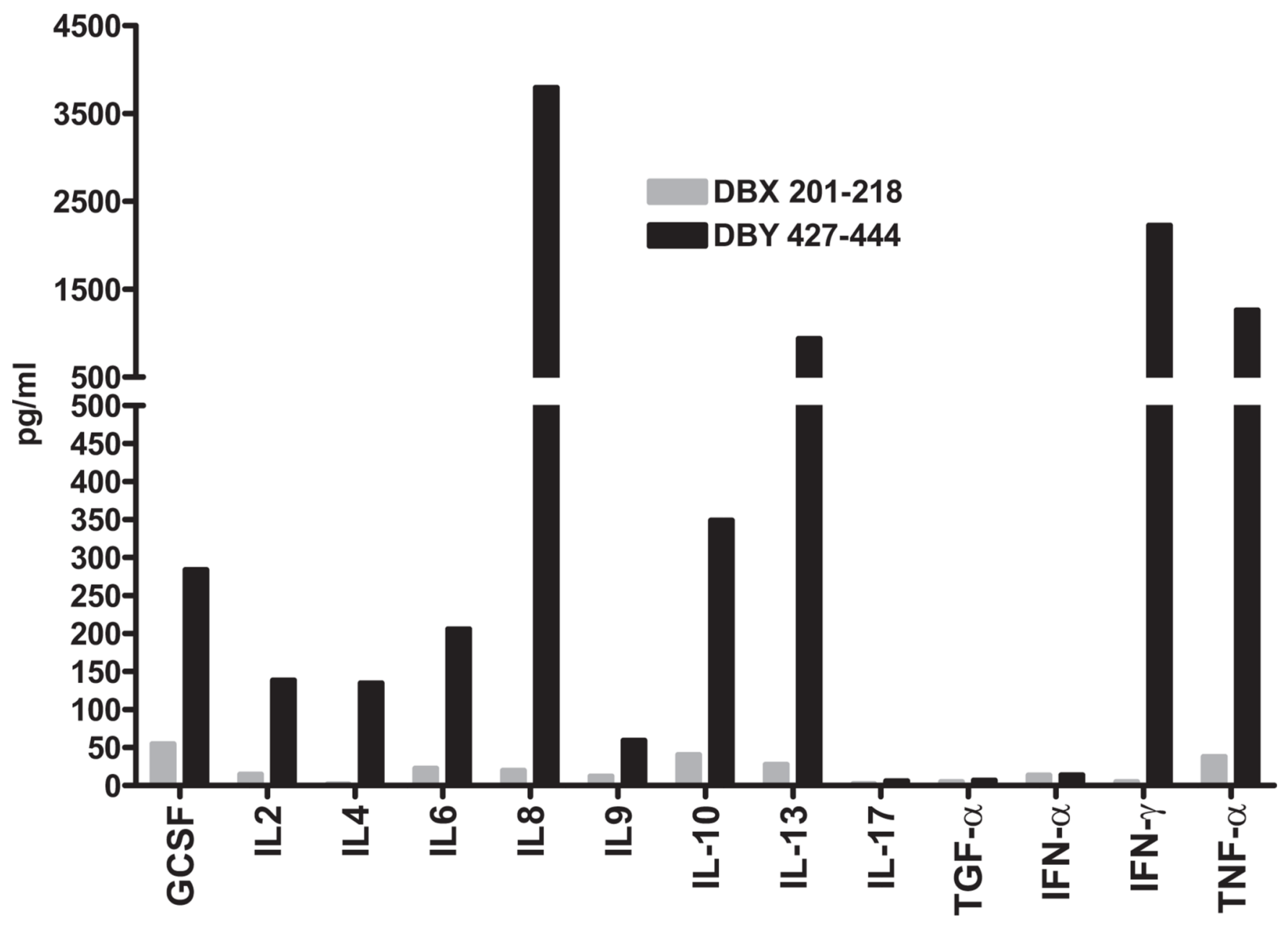

FIGURE 4.

Clone 42 cytokine secretion profile upon stimulation by DBY 427-444. Clone 42 was stimulated with DBY 427-444 or a control peptide, DBX 201-218, pulsed on autologous Epstein-Barr virus (EBV)-B cells. The secretion of different cytokines was measured in the culture supernatant using a multiplex ELISA assay. 

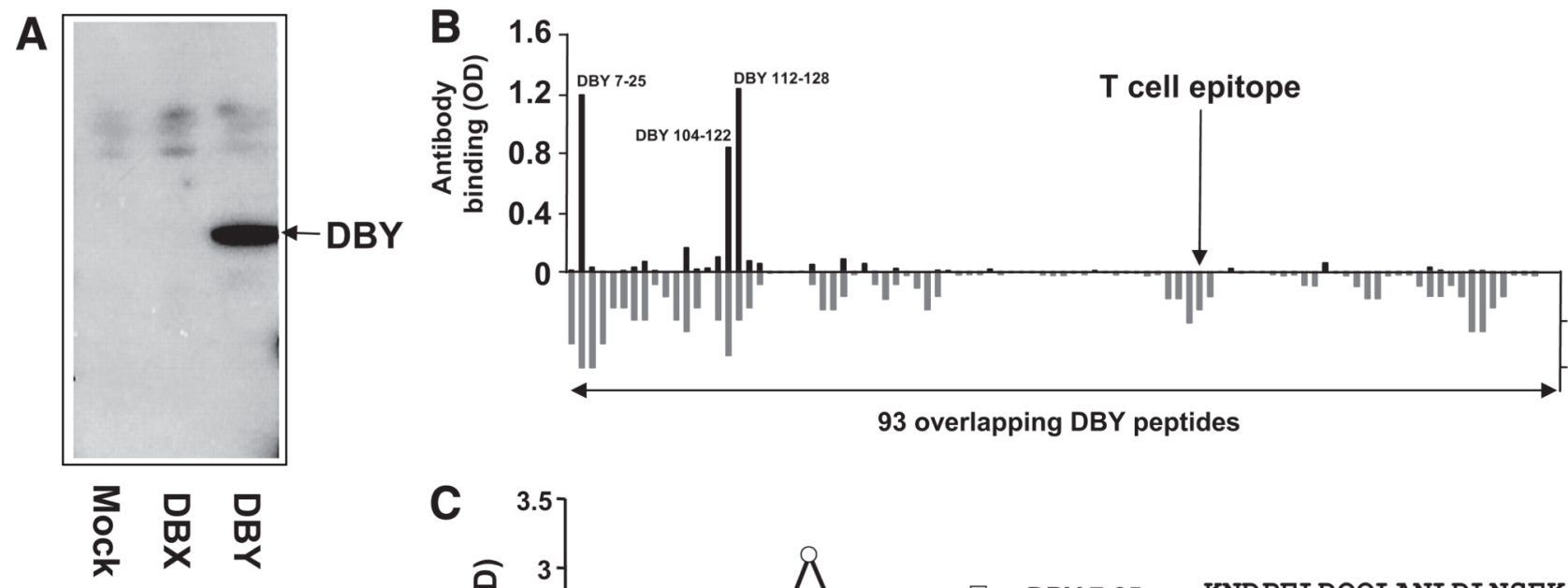

93 overlapping DBY peptides

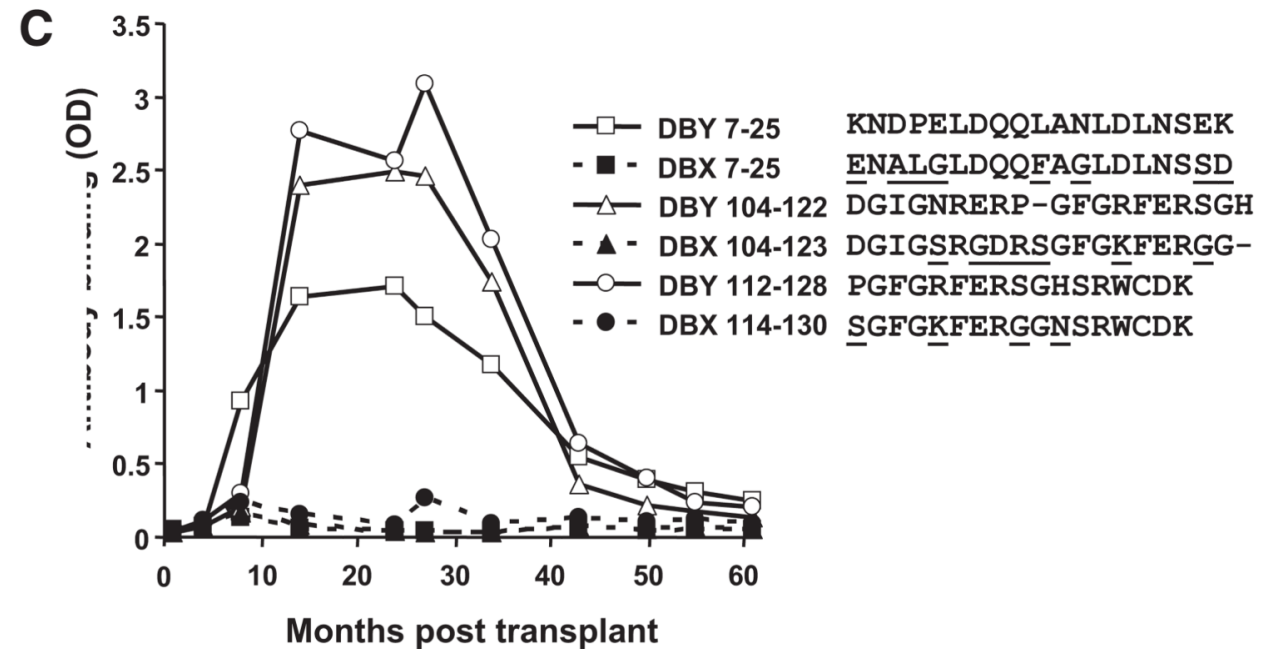

FIGURE 5.

Serologic IgG response to DBY but not DBX after hematopoietic stem-cell transplantation (HSCT). (A). Recombinant DBY and DBX were expressed in human female 293 cells, and total cell lysates were probed in Western blots with plasma collected at 24 months posttransplant. Results with DBY and DBX are compared with 293 cells transfected with an empty vector (mock transfectants). (B). A plasma sample collected from the patient 34 months after transplant was used to map the DBY-specific antibody response. The plasma was diluted 1:50 and tested by ELISA for its reactivity to each of the 93 overlapping DBY peptides (above $x$-axis). Reactivity to the DBY peptides was revealed with anti-IgG secondary antibodies. The location of the T-cell epitope is indicated by an arrow. The number of disparate amino acids between the DBY and peptides and their DBX counterparts is also represented in this figure (below $x$-axis). (C). The patient plasma reactivity to the three most immunogenic DBY peptides, 7-25, 104-122 and 112-128, and their DBX homologues was assessed by ELISA using samples collected serially after allogeneic HSCT. Disparate residues between DBX and DBY peptides are represented with underlined characters. 